AIAA 98-0345

\author{
Open Architecture Data System \\ for NASA Langley Combined Loads \\ Test System
}

\author{
by \\ M. C. Lightfoot and D. R. Ambur \\ NASA Langley Research Center \\ Hampton, VA 23681-0001
}

36th AIAA Aerospace Sciences Meeting \& Exhibit

\author{
January 12-15, 1998 \\ Reno, Nevada
}


AIAA-98-0345

\title{
OPEN ARCHITECTURE DATA SYSTEM FOR NASA LANGLEY COMBINED LOADS TEST SYSTEM
}

\author{
Michael C. Lightfoot ${ }^{1}$ and Damodar R. Ambur ${ }^{2}$ \\ NASA Langley Research Center \\ Hampton, VA 23681-0001
}

\begin{abstract}
The Combined Loads Test System (COLTS) is a new structures test complex that is being developed at NASA Langley Research Center (LaRC) to test large curved panels and cylindrical shell structures. These structural components are representative of aircraft fuselage sections of subsonic and supersonic transport aircraft and cryogenic tank structures of reusable launch vehicles. Test structures are subjected to combined loading conditions that simulate realistic flight load conditions. The facility consists of two pressure-box test machines and one combined loads test machine. Each test machine possesses a unique set of requirements for research data acquisition and real-time data display. Given the complex nature of the mechanical and thermal loads to be applied to the various research test articles, each data system has been designed with connectivity attributes that support both data acquisition and data management functions. This paper addresses the research driven data acquisition requirements for each test machine and demonstrates how an open architecture data system design not only meets those needs but provides robust data sharing between data systems including the various control systems
\end{abstract}

Data System Engineer, Data Systems and Instrument Support Branch.

${ }^{2}$ Assistant Head, Structural Mechanics Branch, Associate Fellow, AIAA.

Copyright (C) 1998 by the American Institute of Aeronautics, Inc. No copyright asserted in the United States under Title 17, U.S. Code. The U.S. Government has a royalty-free license to exercise all rights under the copyright claimed herein the Government Purposes. All other rights are reserved by the copyright owner. which apply spectra of mechanical and thermal loading profiles.

\section{Introduction}

Successful development of viable structures for the wing and fuselage of the advanced subsonic transport and future High-Speed Civil Transport (HSCT) aircraft requires verified structures technology and structural concepts that are both light weight and cost effective. Structural designs and structural analysis for these structures of complex geometry need to be verified by testing appropriate structural elements, structural details, panel subcomponents and built-up structural components that are subjected to realistic loading conditions that are, in general, very complex. The loading conditions for the subsonic and supersonic aircraft include combinations of mechanical, internal pressure and thermal loads, which are cyclic in nature. These tests are needed to identify response characteristics and failure mechanisms that must be accounted for in the structural design process. The tests are also needed to evaluate structural performance and critical design requirements such as damage tolerance and damage containment for the wing and fuselage structures of both subsonic and supersonic aircraft. Pressure containment in fuselage structures is crucial for these passenger aircraft, particularly in the case of the HSCT since this aircraft will cruise at altitudes of about 60,000 $70,000 \mathrm{ft}$., which is twice that for subsonic aircraft. Sudden loss of cabin pressure due to structural failure at these altitudes could be fatal to the passengers and crew.

Some of the important issues that must be addressed for future airframes are pressure containment and the effect of interaction of pressure and mechanical loads on structural 
behavior; buckling and postbuckling response; the effects of cutouts on stability and strength related responses; the effects of joints between components; the interaction of contiguous subcomponents; structural integrity; damage tolerance; and residual strength of metallic and composite structures with damage when subjected to combined loading conditions. The goal for the next five to ten years is to develop stiffness and strength-tailored structures technologies that will enable NASA to be pioneers and leaders in both analytical and experimental structural mechanics areas and support airframe industry with technologies to design and certify future advanced aircraft.

\section{Combined Loads Test System (COLTS)}

Structural testing capability is currently being developed at NASA Langley Research Center to test large curved panels and cylindrical structures of up to 15-ft diameter that are representative of subsonic and supersonic aircraft fuselage structures and launch vehicle cryogenic tank structures while under combined loading conditions. The Combined Loads Test System (COLTS) consists of two pressure-box test machines and one combined loads test machine. The first pressure-box test machine was developed under the Advanced Composite Technology (ACT) Program and is located in B1161 test area and is currently being used for applying biaxial tensile loads to curved panels at room temperature conditions using internal pneumatic pressurization. The second pressurebox test machine is currently undergoing final stages of site installation and will be available for scheduled testing during the first quarter of 1998 to support the Reusable Launch Vehicle (RLV) and High-Speed Research (HSR) Programs. When the second pressure box becomes fully operational it will be capable of subjecting curved panels to combinations of biaxial tension, internal pressure and thermal load conditions consisting of combined states of cryogenic and elevated temperatures that are representative of RLV cryogenic tank structures and HSR fuselage structures. The control and data acquisition systems that support these test machines are currently located at LaRC's B1161 test site in an enclosure constructed within a nearby concrete test cell. Figure 1 shows an aerial view of the B1161 test site with three blast containment test cells and the two pressure-box test frames located adjacent to the concrete embankments.

The third test machine, which was funded by the Advanced Subsonics Technology Programs and HSR Program, is currently being assembled in the B1256 test area and will become operational in March 1998. This third and final test machine in the Combined Loads Test System is a test machine capable of subjecting large cylindrical structures and curved panels under combined states of axial compression or tension, bending moment, torsional shear, internal pressure and elevated temperature conditions. Figure 2 is an aerial view of B1256 Combined Loads test machine site.

\section{Open Architecture Data Acquisition Systems}

The data acquisition systems that support the COLTS test machines are of one generic open architecture design which supports reuse and sharing of both system hardware and software components between sites. Figure 3 outlines the basic design of the data acquisition system. Transducer signals are connected to the Series 300 units for signal conditioning, as needed. The output voltages are then fed into the System 620 data acquisition units where analog-to-digital conversion occurs. As the Series 620 unit buffers data, communications between it and the open architecture computer occurs such that the data are captured, recorded, converted and finally displayed on $X$ terminal displays in several preselected graphical formats.

The hardware front-end system is a Neff Instruments Corporation System 620 scanning analog-to-digital system which is digitally interfaced to a ModComp RealStar 2000 computer system by a 16-bit parallel interface. The RealStar 2000 system consists of a Motorola 88100 RISC processor on a single VME bus CPU board in dual or either quad processor configuration with 80 Megabytes of memory onboard, 4 Gigabytes of attached fixed disk storage, one 1/4" tape drive, a $4 \mathrm{~mm}$ DAT tape drive and a 400 Megabyte removable hard disk. The RealStar computer with its Motorola 88100 processors runs under ModComp's real-time operating system, Real/ix; a variant of the UNIX operating system which has been rewritten for deterministic system performance. While the front-end hardware is typically configured to continuously scan each channel of transducer information, the RealStar 
computer asynchronously receives and services interrupts from the front-end which insures that all data is captured and recorded on the computer's hard disk. As the data recording is being accomplished, engineering unit calculations are performed and made available in tabular engineering unit display formats as periodically required by the operators. In addition to data acquisition setup and control functions, and tabular engineering unit data display management, each data acquisition system includes software for the presentation of real-time two-dimensional graphical displays in engineering units. With this software subsystem in place, operators and test engineers can quickly and intuitively evaluate not only the test procedures but also the performance of the test specimen as loads are quasi-statically applied. The integration of each data acquisition system component was made possible by each vendor using industry standard interfaces for hardware and software. This reduced the amount of custom hardware and software required to fully integrate each system component. Industry standard hardware interfaces such as VME, SCSI, and Ethernet were crucial for system hardware connectivity; while software interface standards such as The $X$ Windows System, C, $C_{++}$, and FORTRAN provided migration paths for earlier versions of code to be moved from legacy systems to open architecture systems designs.

\section{Room Temperature Pressure-Box Data Acquisition System}

The room temperature pressure-box test machine consists of a mechanical test frame with hydraulic load actuators and a pressurized box cavity that is used to uniformly apply biaxial tension to curved panels. The open architecture data acquisition system installed at this site performs continuous data collection and recording of strains, pressures and loads while a pressure and load profile is being quasi-statically applied to a curved panel under test. The system has a maximum channel input capacity of 384 strain gage or bridge-conditioned channels, plus 128 unconditioned channels of transducers signal data. This large channel capacity can easily accommodate the high volume data collection needs for heavily instrumented panels made of composite materials which may possess intentional damage and repaired configurations. Tests on panels such as these not only allow an investigation of damage tolerance but also an evaluation of repair techniques for this Structures technology.

\section{Cryogenic Pressure Box Data Acquisition System}

The cryogenic pressure-box test machine has the same functional construction as the room temperature test machine but has added capability for cryogenic cooling from within the pressure cavity while simultaneously providing elevated temperature heating to the exterior surface of the pressurized test panel. The same data acquisition system used for testing in the room temperature pressure-box is used to support testing in the cryogenic pressure-box testing. By manually switching a set of input cables, the data acquisition system hardware is made available for either room temperature or cryogenic test machines. Given the environment and methods of heating a pressurized panel, additional safety was required to protect the data acquisition system in the event of catastrophic failure resulting in a high-voltage exposure to all instrumentation. To prevent any potential damage to the data acquisition system front-end equipment or open architecture computer system, a series of high-voltage protection modules were designed, developed and installed in series with the data acquisition system. These modules were designed to suppress a high-voltage signal up to 480 volts $A C$ by limiting input voltage and current flow until inline fuses have an opportunity to fail. When using the data acquisition system for cryogenic pressure-box support, full room temperature pressure-box capability is available; up to 384 strain gage or bridgeconditioned channels, and up to 128 thermocouple or unconditioned channels of transducer signal data. This large channel capacity for thermocouples is essential for an evaluation of inplane and through-the-thickness thermal gradients of a candidate RLV or HSR panel with installed thermal protection systems.

Combined Loads Test Machine Data Acquisition System

Figure 4 depicts the configuration of load introduction and load reaction platens for a cylindrical shell as mounted in the Combined Loads Test Machine. This facility has been designed to accommodate cylindrical shells of up to 15 feet in diameter with a maximum length of 45 feet. In addition, a D-Box test fixture can also be inserted between the test machine platens to 
enable combined loads testing of curved panels. There are a total of 10 hydraulic actuators that have been designed to introduce combinations of mechanical loads; up to 2,700 kips in axial load, 600 kips in shear load and 2,900 foot-kips in torsion. A specially designed load control system has been developed and installed to introduce combined load spectra to test articles while simultaneously correcting for unwanted secondary loads. The data acquisition system for this site is the same basic configuration as the pressure-box system but with additional features for enhanced data collection and data sharing. The data acquisition system has a maximum channel input capacity of 512 strain gage or bridge-conditioned channels and $\mathbf{5 1 2}$ thermocouples or unconditioned channels for transducer signal inputs. One additional requirement for this data acquisition system is that it must periodically communicate with the load control system to pass acquired research data values. This transfer of research data will permit the load control system to verify that the test article is experiencing the correct loads as programmed from the load control system. Since the load control system and data acquisition system are two separate systems, a high-speed communications link was required to enable fast transfer of data between the systems. A reflective memory network was established using a product called SCRAMNet from Systran Corp. This technology provides a deterministic means of making shared data available to all nodes on a fiber-optic network without the penalty of each computer managing its communications traffic. Figure 5 depicts the computer systems connectivity using reflective memory and standard Ethernet communications.

\section{High Speed Data Acquisition System}

A high-speed data acquisition system was designed as a roving system to support special tests that require the collection of dynamic or transient data. Transient data could be from tests where structures are subjected to low-speed and high-speed impact and from tests where damage growth occurs catastrophically. The system designed and developed to meet these special test needs consists of a Neff Instruments Corporation System 495 transient data recorder which has been interfaced to a DEC Model $3000 / 500$ workstation running Digital UNIX. Software developed on the DEC computer provides a graphical interface for setup, transfer, storage and display of transient data as captured by the System 495 unit. The system has currently configured supports 32 conditioned channels of transducer data that can be recorded at up to 1 Megahertz sampling rate for up to 2 seconds in duration. Each channel has an analog-to-digital circuit plus memory to hold sampled values. A central system clock controls all channels and therefore, all data are simultaneously acquired upon receiving a triggered event. After a triggered event, data is transferred from the System $\mathbf{4 9 5}$ unit via the SCSI interface, which is a common interface between the DEC workstation and the System 495 unit. Once data is received via the DEC workstation, engineering unit conversions are performed and data are graphically displayed to provide the operator with a visual verification of the quality of the test. With operator concurrence, the data are then stored to disk for later transfer and subsequent archival to another computer via the local area network.

\section{Accomplishments}

The open architecture data acquisition system supporting the Room Temperature Pressure Box has been operational since February 1996. The operators and test engineers of that facility report that they are highly pleased with the overall systems performance. In fact, that facility exceeded all planned test objectives for fiscal year 1997 has received an outstanding rating from within NASA and its industry partners.

The Cryogenic Pressure Box is currently undergoing facility verification testing and control system tuning, therefore no data is available concerning the utilization of the data acquisition system for its combined cryogenic and elevated temperature environment.

The combined loads test machine data acquisition system has currently been installed and is undergoing facility verification testing, along with load control system tuning, in preparation for a March 1998 scheduled test. Since this data acquisition system is identical in design to the others, we anticipate no problems with basic data acquisition and display functions. For example, most graphical displays have been pre-built while the system was temporarily used to support other facilities and identical to the display requirements for the Combined Loads Test Machine. The only new functions that need to be verified are the communications between the data acquisition 
system and the load control system via the reflective memory network.

The high-speed data acquisition system has been installed in a ruggedized environmental enclosure to enable portability between test machines, as required for testing. It has been in use since March 1995 and has been instrumental in permitting the capture of transient strain and displacement data resulting from projectile impact on composite structures.

\section{Future Plans}

Several systems enhancements are required to keep pace with future demands for on-line data display when supporting the combined loads test machine. Figure 5 included two additional systems connected to the reflective memory network. The first system is the temperature control system which will be required to control thermal profiles when a shell section or curved panels requires internal or external elevated temperature exposure. Construction of a heater array is currently underway for a HSR shell that will be externally heated to 350 degrees Fahrenheit as part of a combined loads test. When this system is placed on the reflective memory network, information concerning the performance of heaters and the resulting temperature uniformity will be made available to systems so that test operators may respond appropriately.

Given the complex variety of data to be displayed from each computer system, a Facility Graphics Display Control System is being planned. This system will acquire selected data from each control and data acquisition system and display it using advanced two-dimensional and threedimensional graphics displays.e objective is to increase an operator's ability to evaluate the quality of a test as a test is being conducted.

Also the High Speed Data Acquisition System will undergo a logic board upgrade to increase the system's ability to capture and manage up to eight triggered test events involving a variety of transient and quasi-static events that may occur in a single structures test. Currently only one triggered event is permitted and all data from that event must be saved to disk before another event can be recorded. This planned enhancement will better tailor the data acquisition system to the requirements for future structures testing. 


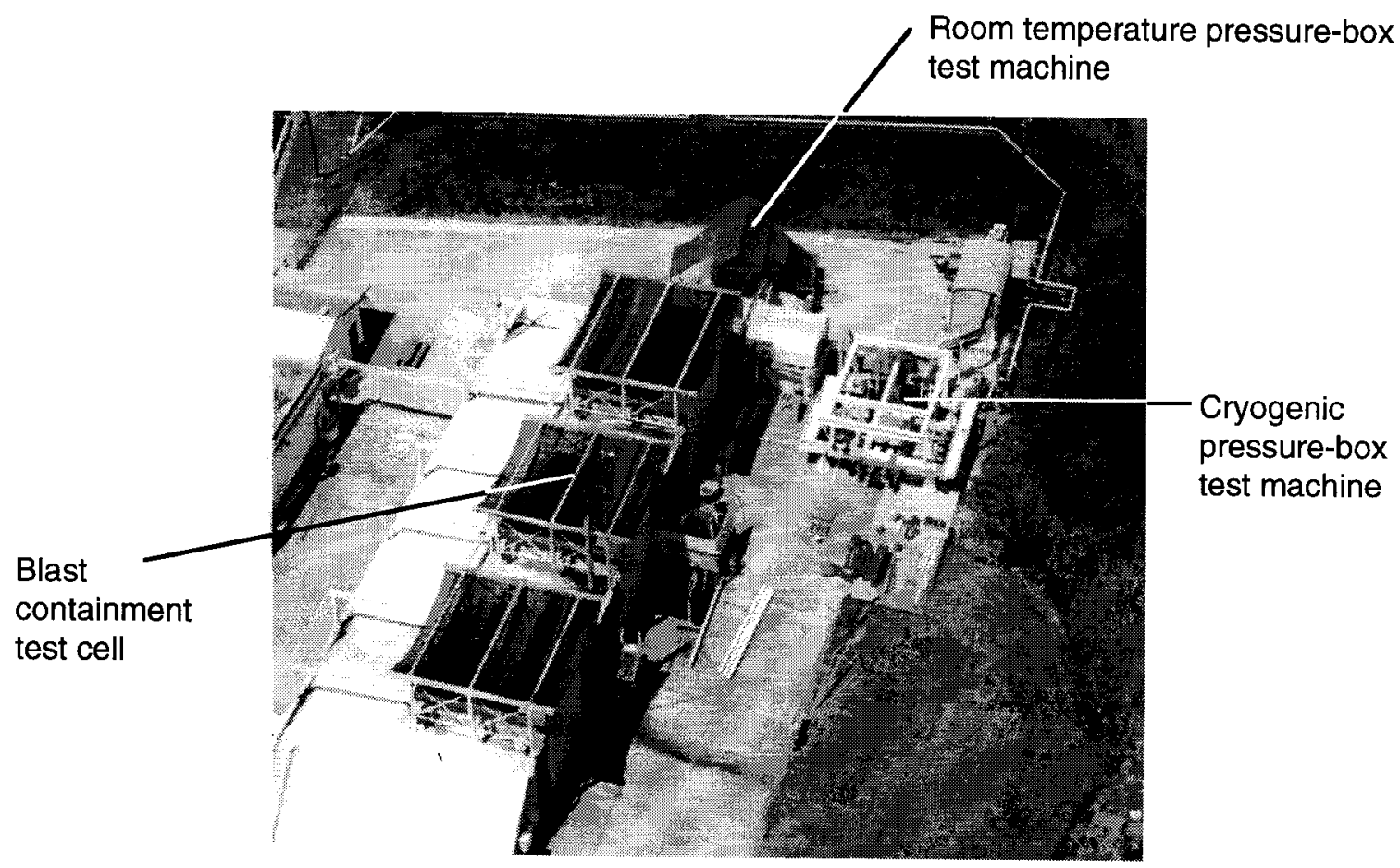

Figure 1. Aerial view of B1161 pressure-box test facility.

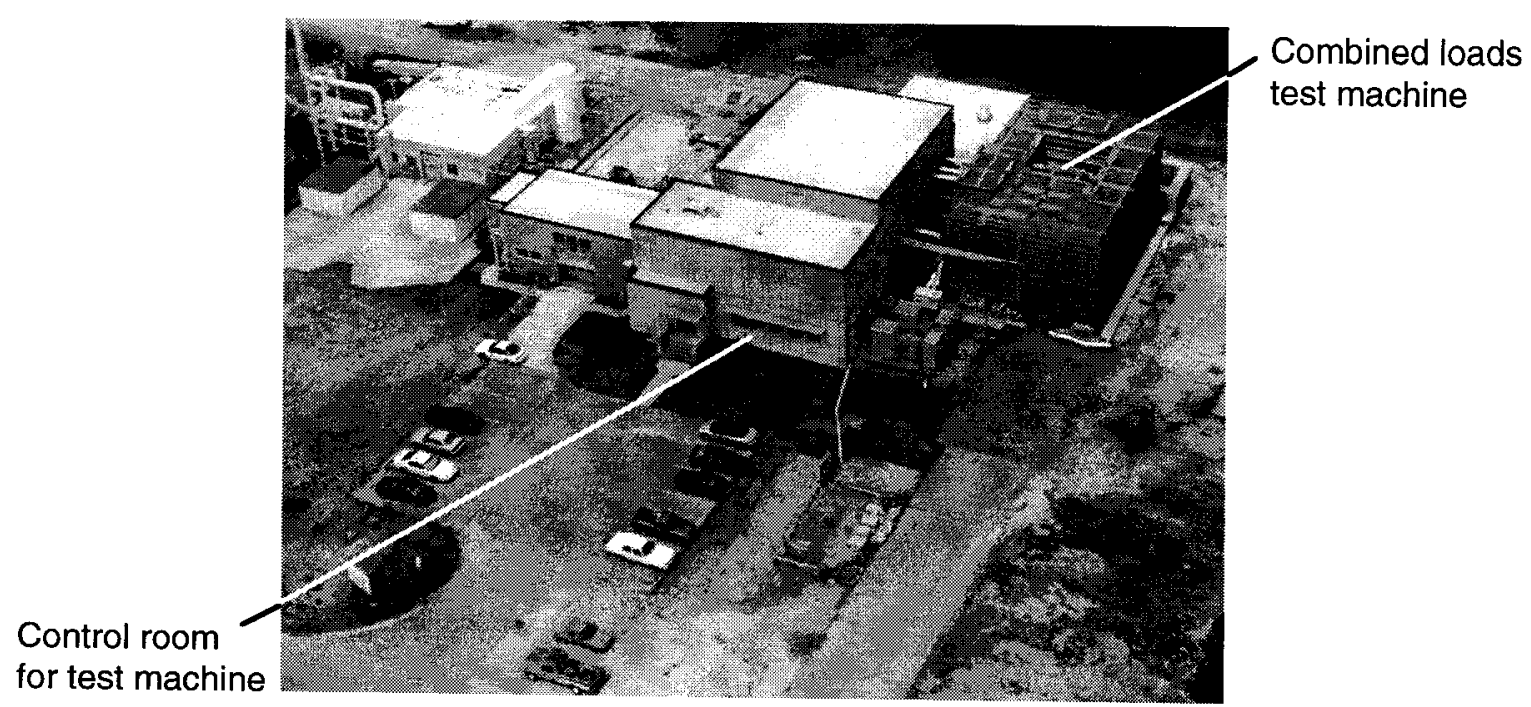

Figure 2. Aerial view of B1256 combined loads test facility. 


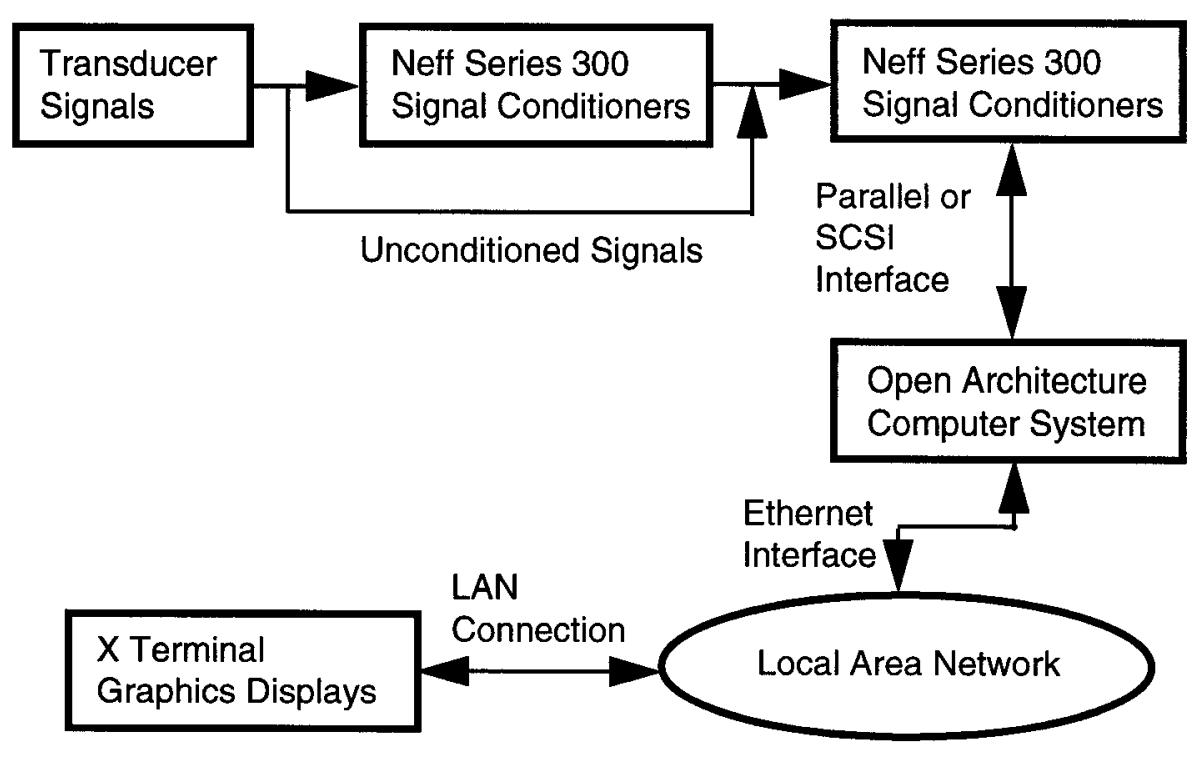

Figure 3. Basic open architecture data acquisition system design.

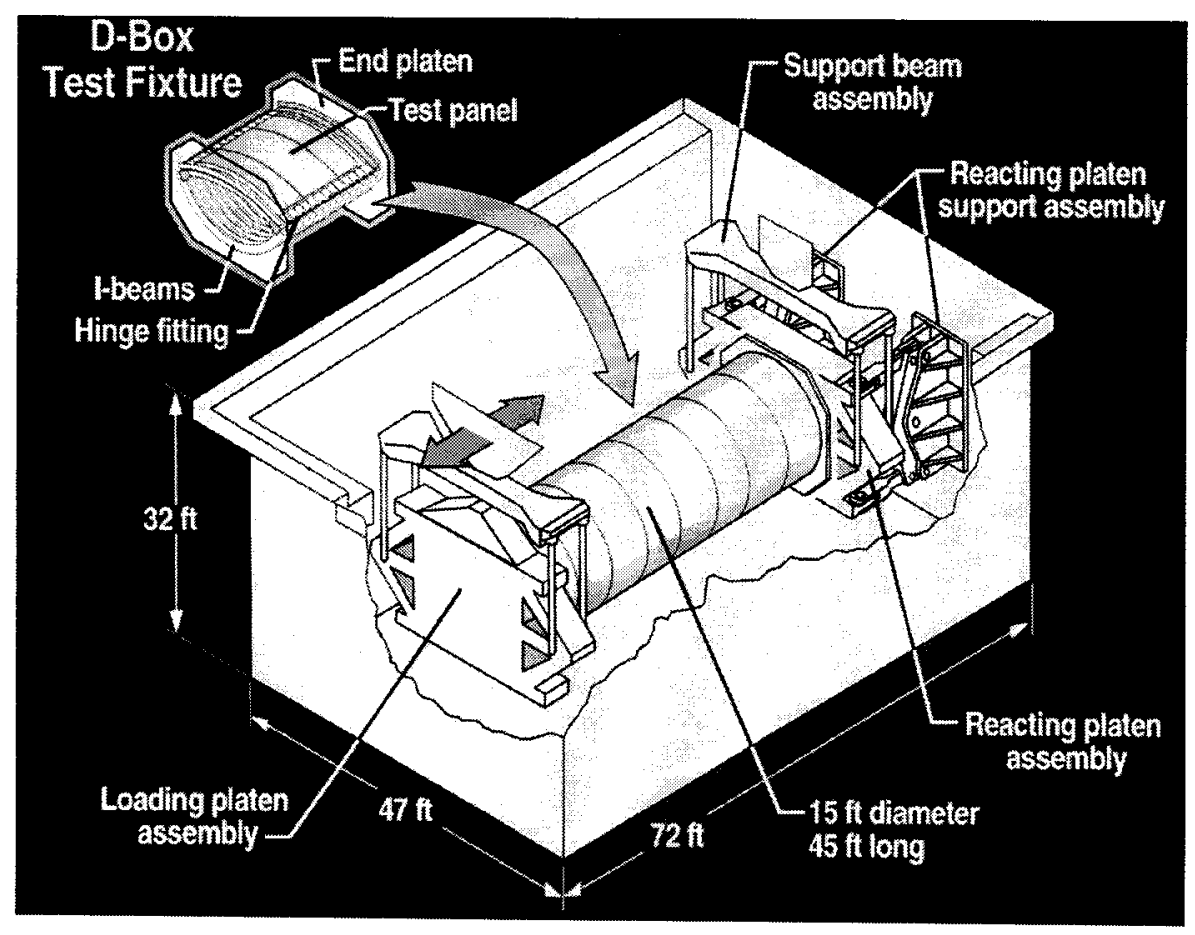

Figure 4. Combined loads test machine design for B1256. 


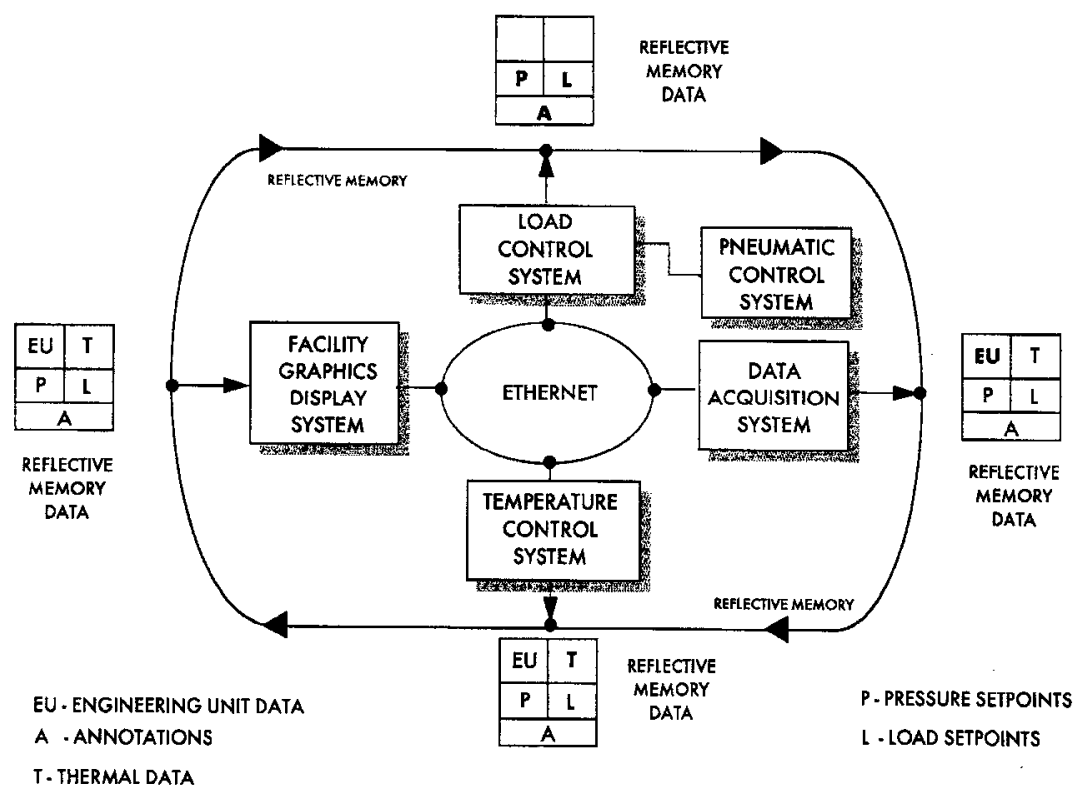

Figure 5. COLTS computer systems connectivity and data sharing. 